\title{
26090 - ULTRASOUND LOCALIZATION IMPROVES PRIMARY SUCCESS AND REDUCES SECONDARY CATHETER FAILURE IN CONTINUOUS PERIPHERAL NERVE BLOCKS
}

\author{
Shalini Dhir MD, Sugantha Ganapathy, FRCPC, \\ University Of Western Ontario, London, ONTARIO, Canada
}

Introduction:

Secondary catheter failure occurs in as high as $40 \%$ of continuous peripheral nerve blocks(1). Ultrasound imaging facilitates brachial plexus localization(2)and is known to improve the quality of blocks and onset times(3). We studied three different techniques of infraclavicular approach to brachial plexus block in terms of efficacy, ease of insertion, onset times and primary and secondary catheter failure rates.

Material and Methods:

60 patients who underwent upper limb surgery under regional block took part in this prospective randomized study. Group TR $(n-=20)$ had needle and catheter inserted by traditional coracoid approach without using the catheter for stimulation. Group ST $(n=20)$ had needle and catheter localization confirmed by stimulation. Group US $(n=20)$ had needle and catheter inserted by ultrasound guidance. In this group, catheter placement was confirmed by agitated 5\% dextrose which was visualized using color doppler with the ultrasound. Nerve stimulation was used to confirm needle tip localization in all groups. All catheters were stimulating variety. Onset of block was defined as complete loss of motor and sensory function in the brachial plexus distribution and was evaluated over a 30-minute period. Incomplete blocks were supplemented after 30 minutes or given general anesthesia. After the block had worn off postoperatively, attempt was made to stimulate the catheter. Local anesthetic was injected via the catheter in an attempt to reestablish the block and the spread was observed under ultrasound imaging.

Results:

Demographics were similar in all three groups. Quality of axillary nerve block was inferior to that in other nerve territories in TR group but it was blocked completely in US group. Secondary catheter failure rate was $11.1 \%$ in US group, $17.6 \%$ in ST group and $84 \%$ in the TR group (chi square $\mathrm{p}<.0001$ ). Post operatively, $77.78 \%$ of US group and $76.47 \%$ of ST group showed adequate spread around the axillary artery. In the TR group, only $26.31 \%$ showed adequate spread around the artery (chi square $\mathrm{p}=.0014$ ). $50 \%$ of patients in TR had bleeding at insertion site that needed compression as compared to $15.8 \%$ in US group. No other complications occurred in any group.

Conclusion:

We conclude that ultrasound guided infraclavicular block provides a rapid and more complete primary block than traditional or stimulating catheter placement. Incidence of GA \&/or supplementation is significantly higher in the non ultrasound guided blocks. Use of ultrasonography for catheter placement reduces secondary catheter failure rate significantly and this is important for postoperative analgesia. Reduced vessel puncture in ultrasound guided placements may obviate vascular complications such as compartment syndrome and neurological sequelae.

References:

1.Salinas F. Editorial. Reg Anesth Pain Med.2003;28:263-72. 
2.Reed J. Anesth Intensive care.1994 Aug;22(4):499

3.Marhofer P. Anesth Analg.1997 Oct;85(4)854-7

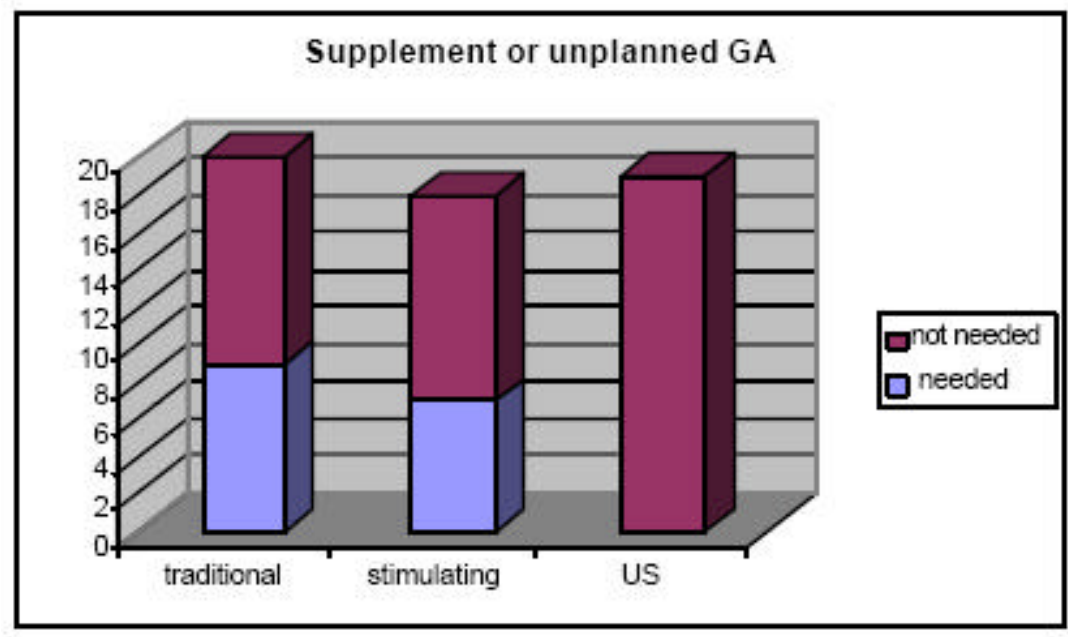

